\title{
BMJ Open Risk factors associated with day-30 mortality in patients over 60 years old admitted in ICU for severe COVID-19: the Senior-COVID-Rea Multicentre Survey protocol
}

Claire Falandry (D) ,1,2 Amélie Malapert, ${ }^{3}$ Mélanie Roche, ${ }^{3}$ Fabien Subtil, ${ }^{4,5}$ Julien Berthiller, ${ }^{6,7}$ Camille Boin, ${ }^{8}$ Justine Dubreuil, ${ }^{9}$ Christine Ravot, ${ }^{1}$ Laurent Bitker, ${ }^{10,11}$ Paul Abraham, ${ }^{12}$ Vincent Collange, ${ }^{13}$ Baptiste Balança, ${ }^{14,15}$ Sylvie Goutte, ${ }^{16}$ Céline Guichon, ${ }^{17,18}$ Emilie Gadea, ${ }^{19}$ Laurent Argaud, ${ }^{20,21}$ David Dayde, ${ }^{3}$ Laurent Jallades, ${ }^{22}$ Alain Lepape, ${ }^{23,24}$ Jean-Baptiste Pialat, ${ }^{25,26}$ Arnaud Friggeri, ${ }^{23}$ Fabrice Thiollière, ${ }^{23}$ Senior-COVID-Rea study Group

To cite: Falandry C, Malapert A, Roche M, et al. Risk factors associated with day- 30 mortality in patients over 60 years old admitted in ICU for severe COVID-19: the Senior-COVID-Rea Multicentre Survey protocol. BMJ Open 2021;11:e044449. doi:10.1136/ bmjopen-2020-044449

- Prepublication history and supplemental material for this paper is available online. To view these files, please visit the journal online (http://dx.doi. org/10.1136/bmjopen-2020044449).

Received 04 September 2020 Accepted 24 April 2021

Check for updates

(C) Author(s) (or their employer(s)) 2021. Re-use permitted under CC BY-NC. No commercial re-use. See rights and permissions. Published by BMJ.

For numbered affiliations see end of article.

Correspondence to

Dr Claire Falandry;

claire.falandry@chu-lyon.fr

\section{ABSTRACT}

Introduction With the spread of COVID-19 epidemic, health plans must be adapted continuously. There is an urgent need to define the best care courses of patients with COVID-19, especially in intensive care units (ICUs), according to their individualised benefit/risk ratio. Since older age is associated with poorer short-term and longterm outcomes, prediction models are needed, that may assist clinicians in their ICU admission decision. SeniorCOVID-Rea was designed to evaluate, in patients over 60 years old admitted in ICU for severe COVID-19 disease, the impact of age and geriatric and paraclinical parameters on their mortality 30 days after ICU admission.

Methods and analysis This is a multicentre survey protocol to be conducted in seven hospitals of the Auvergne-Rhône-Alpes region, France. All patients over 60 years old admitted in ICU for severe COVID-19 infection (or their legally acceptable representative) will be proposed to enter the study and to fill in a questionnaire regarding their functional and nutritional parameters 1 month before COVID-19 infection. Paraclinical parameters at ICU admission will be collected: Iymphocytes and neutrophils counts, high-fluorescent lymphoid cells and immature granulocytes percentages (Sysmex data), D-dimers, Creactive protein, lactate dehydrogenase (LDH), creatinine, CT scan for lung extension rate as well as clinical resuscitation scores, and the delay between the first signs of infection and ICU admission. The primary outcome will be the overall survival at day 30 post-ICU admission. The analysis of factors predicting mortality at day 30 will be carried out using univariate and multivariate logistic regressions. Multivariate logistic regression will consider up to 15 factors.

The ambition of this trial, which takes into account the different approaches of geriatric vulnerability, is to define the respective abilities of different operational criteria of frailty to predict patients' outcomes.

Ethics and dissemination The study protocol was ethically approved. The results of the primary and
Strengths and limitations of this study

- This study will provide a cross-sectional analysis of the impact of geriatric parameters, and particularly frailty according to Clinical Frailty Score and Fried's criteria, on mortality at day 30 after intensive care unit (ICU) admission.

- This study will provide critical information regarding the personalised benefit/risk balance of ICU admission in patients over 60 years old.

Broad inclusion criteria and systematic screening will limit inclusion biases.

- As knowledge and techniques regarding the management of patients with COVID-19 change rapidly, mortality and ICU capacities may differ over time.

- Differences in COVID-19 management may also differ depending on the different ICU teams.

secondary objectives will be published in peer-reviewed journals.

Trial registration number NCT04422340.

\section{INTRODUCTION}

Since the outbreak of the COVID-19 epidemic (2019, Wuhan, China), health plans have been continuously adapted in response to the emergency of the sanitary situation, especially regarding the intensive care units (ICUs) capacities. The first studies were based on data from the Chinese population and demonstrated a more elevated incidence of COVID-19 infections in older people $(\geq 60$ years old) compared with younger people, as well as a higher frequency of severe forms of the disease ${ }^{1}$ and, therefore, more theoretical indications of ICU admission. 
However, the first published data from the Hubei province suggested a low benefit of resuscitation for patients between 70 years and 80 years old and a null benefit for patients over 80 years old. ${ }^{1}$ More recent data from Lombardy, Italy, reported a $41 \%$ mortality rate in patients over 70 years old, $55 \%$ in patients over 80 years old and $100 \%$ in patients over 90 years old, even though they admitted in ICU. ${ }^{2}$ Consequently, the individual benefit/ risk balance of an orientation towards resuscitation for each age category should be considered in terms of quality of life and taking into account the limit unreasonable obstinacy.

So far, cardiovascular comorbidities, some laboratory parameters (lactate dehydrogenase $(\mathrm{LDH}),{ }^{3}$ creatinine, lymphocytes, neutrophils, prothrombin time, D-dimers, ${ }^{4}$ etc), and the time between the symptom onset and the entry into resuscitation have been identified as covariates associated with resuscitation mortality. Cumulative evidence highlights the interest of some additional biological parameters extracted from extended complete blood cell count under fluorescence flow cytometry $\left(\right.$ Sysmex data ${ }^{5-7}$ ).

Another covariate classically proposed to predict patient outcomes is frailty. Frailty has been defined as a state of increased vulnerability to poor resolution of homoeostasis after a stressor event, which increases the risk of adverse outcomes, including falls, delirium and disability. ${ }^{8}$ Although some consensus has been reached regarding its definition, its operational criteria differ grossly between a multidomain view, according to which frailty is the consequence of the addition of several deficits ${ }^{9}$ and a phenotypical view, according to which prefrail and frail patients develop a specific phenotype related to malnutrition and sarcopenia. ${ }^{10}$ The Frailty Index-a scoring system according the cumulative deficits view of frailtyhas been used in an Italian study from the COVID-19 Monza Team members to discriminate retrospectively the patients who recovered from those who either died or were transferred to ICU. ${ }^{11}$ Such results suggest that the outcomes of patients with COVID-19 admitted to the hospital are better predicted by frailty than either age or comorbidities. The Clinical Frailty Score (CFS) enables the stratification of older patients into distinct levels of fitness using a rapid 'at-a-glance' assessment. ${ }^{12}$ Using this score, the COVID-19 in Older People European cohort study, mainly performed in the UK, was able to correlate frailty with the total mortality and the day-7 mortality in all patients admitted to hospital for COVID-19 infection. ${ }^{13}$ On 20 March 2020, the UK's National Institute for Health and Care Excellence published a COVID-19 Rapid Guideline (critical care). According to it, only patients with a CFS less than 5 should be considered for critical care, ${ }^{14}$ as this threshold was previously shown to predict a higher mortality in (non-COVID) older patients admitted in ICU. $^{15}$

However, the geriatric community promptly reacted to these guidelines, pointing out the risk of drift in assessing the CFS, designed to be performed by trained geriatricians ${ }^{1617}$ and the ethical dilemma of transforming the frailty spectrum into a binary covariate, considering that the inter-rater variability may be high between CFS scores 4 and $5 .{ }^{17}$ Moreover, two studies evaluating the impact of CFS score in COVID-19 versus non-COVID-19 populations stated that CFS is not a good discriminator of prognosis in COVID-19 infected population. ${ }^{18} 19$

Nevertheless, and independently of COVID-19, there has been a growing interest in the specificities of the older population in ICUs, due to generally more deleterious short-term and long-term outcomes and the identification of predictive risk factors that may assist clinicians in their ICU admission decision. ${ }^{1520} 21$

In an intent to better define the individual benefit/risk ratio of ICU admission for each age category of patients, the objective of this multicentre observational study is to determine the clinical and laboratory covariates predictive of mortality among patients with COVID-19 over 60 years old admitted in ICU and depending on their age. A specific attention will be paid to their functional and nutritional parameters (retrospective and declarative assessment) 1 month before infection.

\section{METHODS AND ANALYSIS \\ Objectives}

\section{Primary objective}

Evaluation of the impact of age on mortality at day 30 after admission to ICU.

\section{Secondary objective}

The secondary objectives of the study are (a) evaluation of the impact of the following co-variables on mortality at day 30: comorbidities 1 month prior to infection (grade $\geq 2$, scored on the Cumulative Illness Rating ScaleGeriatric (CIRS-G) ${ }^{22}$ ) and more specifically, cardiac and vascular comorbidities (grade $\geq 2$ on the CIRS-G); the functional status 1 month before infection, assessed by the caregiver using the CFS and the activity of daily living $(\mathrm{ADL})^{23}$ and instrumental $\mathrm{ADL}^{24}$ scores; nutritional data (weight at hospital and ICU admission, weight loss in the last 1 month and 6 months before infection and the presence of mild or severe anorexia); laboratory data at ICU admission (LDH, C-reactive protein (CRP) and creatinine levels, as well as lymphocytes and neutrophils count, and Sysmex data (immature granulocyte count and high fluorescent lymphocyte count $)^{6-8}$ ); chest imaging data (COVID-19 lung extension rated as minimal, moderate, extensive, severe or critical according to the French Radiology Society guidelines ${ }^{25}$ ); and resuscitation parameters at ICU admission (arterial oxygen pressure/fraction of inspired oxygen ratio, indice de gravité simplifié II/ simplified acute physiology score II (IGS II/SASP II ${ }^{26}$ ) and/or sepsis-related organ failure assessment score (a posteriori estimate based on IGS II/SASP $\mathrm{II}^{27}$ ), and delay between the first signs of infection and admission to ICU and (b) evaluation of the impact of age and frailty scores (CFS and Fried's frailty score) on: medical management 
in the ICU and during the total hospital stay; and medical complications in the ICU and during the hospital stay after ICU discharge.

\section{Study design}

Senior-COVID-Rea is a retrospective and prospective multicentre study analysing data from patient charts. Patients were retrospectively included from the starting of COVID-19 pandemic in France (February 2020) to 10 April 2020, and prospectively from 10 April 2020. Data processing has been approved by the Commission nationale de l'informatique et des libertés (French data protection commission).

\section{Study centres and participants}

All patients over 60 years old admitted between February 2020 (the beginning of the COVID-19 outbreak in France) and 7 May $2020^{11}$ in ICUs from the Senior-COVID-Rea investigation centres (Hôpital Lyon Sud, Hôpital de la Croix-Rousse, Hôpital Edouard Herriot and Hôpital Lyon Est from the Hospices Civils de Lyon, and the Médipôle Lyon-Villeurbanne, Hôpital Nord-Ouest Villefranche-surSaône and Hôpital Emile Roux in Le Puy-en-Velay) are eligible for inclusion.

Inclusion criteria are: being aged 60 years or more, being admitted to an ICU and having a COVID-19 diagnosis confirmed by reverse transcriptase-PCR or chest scanner. The sole exclusion criterion is refusal to participate in the study.

Individual information explaining the study will be given to the patients. Depending on their clinical condition, a waiver request is justified in accordance with the ICH (International Council for Harmonisation of technical requirements for pharmaceuticals for human use) harmonised guidelines for clinical practice of 9 November 2016: 'In emergency situations, when prior consent of the subject is not possible, the consent of the subject's legally acceptable representative, if present, should be requested'. In accordance with the regulatory framework, information will be given to the patient's legally acceptable representative. The information leaflet will be given to patients as soon as they are able to read it; in the event of refusal to participate, the patient will be removed from the study.

Each included patient will be assigned an anonymous identification number.

\section{Outcomes and measurements \\ Primary outcome}

The primary outcome is the overall mortality at day 30 after admission in ICU.

\section{Secondary outcomes}

The secondary outcomes of the study are: (a) the mortality in ICU, (b) the overall survival, the length of stay (in the ICU, in total, in a rehabilitation when applicable), (c) the medical management in the ICU (high flow oxygen therapy, non-invasive ventilation, invasive ventilation, prone position, extracorporeal membrane oxygenation, catecholamines, extrarenal purification, intermittent haemodialysis, continuous venovenous haemodiafiltration, short-acting hypnotics, long-acting hypnotics, short-acting analgesics, long-acting analgesics, dexmedetomidine or catapressan, neuroleptic for sedation (levomepromazine and cyamemazine), withholding or withdrawing life-sustaining treatments (specifying the limited intervention(s)), anti-infectious treatments (antibiotherapy lines, remdesivir, lopinavir/ritonavir, hydroxychloroquine, azithromycin and other), corticosteroids, nutrition (parenteral or enteral feeding, and oral nutrition), physiotherapy (passive or active mobilisation, armchair bed transfer, edge of the bed sitting, armchair and verticalisation) and during the total hospital stay (oxygen therapy, anti-infectious treatments (antibiotherapy lines, remdesivir, lopinavir/ritonavir, hydroxychloroquine, azithromycin and other), corticosteroids, nutrition (parenteral or enteral feeding, and oral nutrition), physiotherapy (passive or active mobilisation, and walk)), and (d) the medical complications in the ICU (acquired delirium or confusion, acquired neuropathy, acquired lung infections, catheter-related infections, bacteraemia, pressure sore, necrosis of the extremities, deep vein thrombosis, pulmonary embolism, swallowing disorders, congestion/trouble with ventilation, tracheotomy, joint stiffening, neurological deficit secondary to stroke, peripheral nerve compression, multiple intubations and their count, intrahospital transports and their count) and during the hospital stay after resuscitation (delirium or confusion, postresuscitation neuropathy, acquired lung infection(s), catheter-related infection(s), bacteraemia, pressure sore, necrosis of the extremities, deep vein thrombosis, swallowing disorders, joint stiffening, neurological deficit secondary to stroke and peripheral nerve compression).

\section{Sample size calculation}

To assess the effect of age on mortality in ICU, it is planned to compare mortality between patients under 70 years old and over 70 years old. Based on the results from a Chinese retrospective study, ${ }^{1}$ the hypothesis of the SeniorCOVID-Rea study was the following: considering a single analysis variable (age), and an expected mortality of $30 \%$ for patients under 70 years old and $70 \%$ for patients over 70 years old (patients over 70 years old representing $40 \%$ of the study population), it will be necessary to include a total of 130 patients to show a statistically significant difference between these two groups with a power of $90 \%$ (bilateral alpha risk test of $5 \%$ ). Since the analysis considers the integration of several factors, considering 15 factors, hoping for a coefficient of determination of 0.5 of the model, to achieve an optimism of less than $10 \%$, it will be necessary to include 185 patients (criterion 1 of Riley et $a l^{28}$ ).

After the publication of data on mortality in ICU from Lombardy, Italy, in April 2020, ${ }^{2}$ it was considered that closing the inclusion process after 185 inclusions would impair the statistical power of the study and induce a 
potential patient selection bias. As a consequence, the scientific committee decided on 7 May 2020 that all the patients admitted to ICU before that date-that corresponded to the end of the first COVID-19 wave in France-were eligible and proposed no limitation in terms of number of inclusions for this study. This sample size calculation was modified on Clinicaltrials.gov site accordingly (28 July 2020).

\section{Data management and statistical analyses}

Data are monitored by a clinical research associate (CRA). Inconsistencies will be reported to the study investigators who will decide whether the data should be corrected or considered as missing. All changes in the data will be notified.

The analyses will be carried out by an independent statistician using R (R Core Team; a language and environment for statistical computing; $\mathrm{R}$ Foundation for Statistical Computing, Vienna, Austria; https://www.Rproject.org/).

All the characteristics collected will be subjected to a descriptive analysis.

\section{Descriptive analyses}

A flow-chart diagram will describe the process of patient inclusion and exclusion (refusal to participate and patient's request to terminate participation in the study) during the trial time frame and the characteristics of the patients excluded.

The characteristics of the study population and the proportion of missing values will be reported. Patient characteristics will be expressed as mean $( \pm \mathrm{SD})$ or median (IQR) for quantitative variables and counts and percentages for categorical variables.

\section{Primary analysis}

The analysis of factors predicting mortality at day 30 will be carried out using univariate and multivariate logistic regressions. The multivariate logistic regression will consider up to 15 factors. In view of the results of the univariate analyses and the correlation between the factors studied, it will be decided which factors will be included in the multivariate analyses. Multicollinearity will be analysed by Venn diagram and variance inflation factors. The effect of each factor will be quantified and expressed as the OR and the associated $95 \%$ CI.

\section{Secondary analyses}

The mortality proportion in ICU and the 95\% CI will be calculated for the two groups of patients (dead vs alive, and according to age). The overall survival will be described using Kaplan-Meier survival curves in these two groups; the mean and median length of stay in ICU will also be reported.

The association of age and of the frailty scores (using cut-offs) with the medical management and medical complication outcomes will be quantified and expressed as OR and the associated 95\% CI in an exploratory analysis. In this context, no adjustment will be made for multiple testing.

\section{Data monitoring}

The successful completion of the database is ensured by the hospital CRA. The hospital CRA also ensures the compliance with the study protocol. The sponsor CRA verifies that the rights of the participants are respected.

\section{End of protocol}

According to studies analysing data from patient charts, and according to French regulation, patients leave the study either on a per-protocol basis on day 30 after their admission in intensive care ward or at any time during the conduct of the study if they no longer wish to participate. However, as indicated in the information letter to the patients/caregivers, the data collected before exclusion may be used as part of the study.

\section{Confidentiality}

Correspondence tables will be kept in a separate file that does not contain clinical data. The access to the nominative information is protected by a password and confidentiality is guaranteed.

\section{Protocol amendments}

Any important modifications requiring a new ethics committee approval will be communicated in future publications. Any potential impact of protocol modifications on the results will be discussed as appropriate.

\section{Trial status}

Patient enrolment began on 10 April 2020. Data are being collected.

\section{Patients and public involvement}

Due to COVID-19 emergency and as this trial is health data-based, patients were not involved in the design of the trial. The information notice was written according a model validated by a patients' association (Entrepôt des Données de Santé information notice model).

\section{DISCUSSION}

\section{Discussion of the study design}

This study has an ethical stake in the evaluation of the risk/benefit balance of referring patients over 60 years old to intensive care procedures, according to their comorbidities and pre-infection functional state, as well as to the severity of the pathology at the time of care. Patients over 60 years old appear to be at risk of developing severe forms of COVID-19 infection. The identification of prognostic factors is a major issue for the personalisation of care of these patients.

More than chronological age, frailty appears as a good prognostic marker in response to variable stressors. ${ }^{29}$ Frailty has been previously demonstrated as a good prognostic marker of the ability to overcome resuscitationassociated stress in diverse contexts, ${ }^{30}$ as well as a 
predictive marker of complications during non-invasive mechanical ventilation, ${ }^{31}$ extubation failure, ${ }^{32}$ resources utilisation ${ }^{33}$ and poor functional recovery and long-term quality of life. ${ }^{34}$ Therefore, using frailty as a prognostic marker and as a tool for the adaptation of care management appears logical and ethical, especially in contexts of high stress, such as COVID-19 severe infections.

The ambition of this trial, which takes into account the different approaches of frailty, is to define the respective abilities of different operational criteria of frailty to predict patients' outcomes.

\section{Ethics and dissemination}

The study sponsor is the Hospices Civils de Lyon. In response to the COVID-19 emergency and the regulation on health data, accrual started on 10 April 2020. The study protocol (V.1.0 of 7 April 2020) was approved by an ethics committee (Comité Scientifique et Éthique des Hospices Civils de Lyon) on 30 June 2020 and declared on ClinicalTrials platform on 9 June 2020. The research will be carried out in accordance with the Declaration of Helsinki and ICH Good Clinical Practice Guidelines. Trial protocol fulfils the Standard Protocol Items: Recommendations for Interventional Trials 2013 checklist (online supplemental table 1) and WHO Trial Registration Data Set (online supplemental table 2). The study complies with the principles of the data protection act in France and with the General Data Protection Regulations in force in Europe. Each investigator must collect non-objection from patients and/or their relatives at the beginning of the procedure (Annexes 1-3, in French). This nonobjection is logged in the patient's medical chart. The patient can withdraw his/her consent for participation in the study at any time with an oral information to the investigator or clinical research assistant.

The results of the primary and secondary objectives will be published in peer-reviewed journals. All authors of future publications will have to meet the criteria for authorship stated in the Uniform Requirements for Manuscripts Submitted to Biomedical Journals by the International Committee of Medical Journal Editors.

\section{Author affiliations}

${ }^{1}$ Service de Gériatrie, Centre Hospitaliser Lyon Sud, Hospices Civils de Lyon, PierreBénite, France

'Laboratoire CarMeN, Inserm U1060, INRA U1397, Université Claude Bernard Lyon 1, INSA Lyon, Faculté de Médecine et de Maïeutique Charles Mérieux, Université de Lyon, Oullins, France

${ }^{3}$ Plateforme Transversale de Recherche de I'ICHCL, C, Hospices Civils de Lyon, Pierre-Bénite, France

${ }^{4}$ CNRS UMR5558, Laboratoire de Biométrie et Biologie Evolutive, Université Claude Bernard Lyon 1, Université de Lyon, Villeurbanne, France

${ }^{5}$ Service de Biostatistique, Hospices Civils de Lyon, Lyon, France

${ }^{6}$ Université Claude Bernard Lyon 1 - Domaine de Rockefeller, Lyon, France

${ }^{7}$ SREC - PSP - Cellule innovation, Hospices Civils de Lyon, Bron, France

${ }^{8} \mathrm{DRCl}$, Hospices Civils de Lyon, Lyon, France

${ }^{9}$ Plateforme Transversale de Recherche de l'ICHCL - Groupement Hospitalier Sud, Hospices Civils de Lyon, Pierre-Bénite, France

${ }^{10}$ Service de Réanimation Médicale, Hôpital de La Croix Rousse, Hospices Civils de

Lyon, Lyon, France

${ }^{11}$ CREATIS INSERM 1044 CNRS 5220, Université de Lyon, Lyon, France
${ }^{12}$ Département d'anesthésie-réanimation, Hôpital Edouard-Herriot, Hospices Civils de Lyon, Lyon, France

${ }^{13}$ Département Anesthésie-réanimation, Medipôle Lyon-Villeurbanne, Villeurbanne, France

${ }^{14}$ Département d'anesthésie et réanimation neurologique, Hôpital Pierre Wertheimer, Hospices Civils de Lyon, Lyon, France

${ }^{15}$ Inserm U1028, CNRS UMR 5292, Lyon Neuroscience Research Centre, Team TIGER, University of Lyon, Lyon, France

${ }^{16}$ Service de gériatrie, Hôpital Nord-Ouest, Gleizé, France

${ }^{17}$ Service d'anesthésie - réanimation, Hôpital de la Croix Rousse, Hospices Civils de Lyon, Lyon, France

${ }^{18}$ Université Lyon 1, Université de Lyon, Lyon, France

${ }^{19}$ Département de Recherche Clinique, Centre Hospitalier Emile Roux, Le Puy en Velay, France

${ }^{20}$ Faculté de médecine Lyon-Est, Université Claude Bernard Lyon 1, Université de Lyon, Lyon, France

${ }^{21}$ Service de Médecine Intensive-Réanimation Médicale, Hôpital Edouard Herriot, Hospices Civils de Lyon, Lyon, France

${ }^{22}$ Service d'Hématologie biologique - Groupement Hospitalier Sud, Hospices Civils de Lyon, Pierre-Bénite, France

${ }^{23}$ Intensive Care Unit, Centre Hospitalier Lyon Sud, Hospices Civils de Lyon, Lyon, France

${ }^{24}$ Centre International de Recherche en Infectiologie, Université de Lyon, Lyon, France

${ }^{25}$ Département de Radiologie, Groupement Hospitalier Sud, Hospices Civils de Lyon, Pierre-Bénite, France

${ }^{26}$ CREATIS CNRS UMR 5220 INSERM U1206, Université de Lyon, Lyon, France

Acknowledgements The authors acknowledge the research teams from the Senior-COVID-Rea investigation centres (Hôpital Lyon Sud, Hôpital de la CroixRousse, Hôpital Edouard Herriot and Hôpital Lyon Est from the Hospices Civils de Lyon, and the Médipôle Lyon-Villeurbanne, Hôpital Nord-Ouest Villefranche-surSaône and Hôpital Emile Roux in Le Puy-en-Velay) who contribute to the enrolment of patients in this study. The authors would like to thank patients and their families to contribute to this research despite the urgent and stressful context. They also thank Hélène Boyer (DRCl, Hospices Civils de Lyon) for help in the manuscript preparation.

Collaborators The Senior-COVID-Rea study group: coordinator: Claire Falandry; ICU physicians: Paul Abraham, Laurent Argaud, Baptiste Balança, Laurent Bitker, Arnaud Friggeri, Celine Guichon, Alain Lepape, Christophe Leroy, Anne-Claire Lukaszewicz, Thomas Rimmelé, Marie Simon, Fabrice Thiollière, Charles-Hervé Vacheron and Hodane Yonis; geriatricians: Antoine Garnier-Crussard and Max Haïne; statistician: Fabien Subtil; methodologist: Julien Berthiller; radiologist: Jean-Baptiste Pialat; haematologist: Laurent Jallades; clinical research team: Loredana Baboi, Camille Boin, Valérie Cerro, David Dayde, Justine Dubreuil, Carlos El Khoury, Emilie Gadéa-Deschamps, Marie-Catherine Fromont, Audrey Gelot, Sylvie Goutte, Anthéa Loïez, Amélie Malapert, Céline Monard, Maya Perrou, Laetitia Paradisi-Prieur, Marion Provent, Mélanie Roche, Gulsum Sahin, Ghyslaine Thao and Marine Thieux.

Contributors All authors (CF, AM, MR, FS, JB, CB, JD, CR, LB, PA, VC, BB, SG, CG, EG, LA, DD, LJ, AL, J-BP, AF and FT) participated in the Senior-COVID-Rea protocol conception, critically reviewed and approved the final version of the protocol. CF led the drafting of the manuscript.

Funding This work was supported by the Hospices Civils de Lyon, and was selected through an internal call for 'COVID-19' projects (decision of the selection committee on 12 May 2020). It provided partial funding for statistical analysis and clinical research assistants.

Competing interests None declared.

Patient consent for publication Obtained.

Provenance and peer review Not commissioned; externally peer reviewed.

Supplemental material This content has been supplied by the author(s). It has not been vetted by BMJ Publishing Group Limited (BMJ) and may not have been peer-reviewed. Any opinions or recommendations discussed are solely those of the author(s) and are not endorsed by BMJ. BMJ disclaims all liability and responsibility arising from any reliance placed on the content. Where the content includes any translated material, BMJ does not warrant the accuracy and reliability of the translations (including but not limited to local regulations, clinical guidelines, terminology, drug names and drug dosages), and is not responsible for any error and/or omissions arising from translation and adaptation or otherwise. 
Open access This is an open access article distributed in accordance with the Creative Commons Attribution Non Commercial (CC BY-NC 4.0) license, which permits others to distribute, remix, adapt, build upon this work non-commercially, and license their derivative works on different terms, provided the original work is properly cited, appropriate credit is given, any changes made indicated, and the use is non-commercial. See: http://creativecommons.org/licenses/by-nc/4.0/.

ORCID iD

Claire Falandry http://orcid.org/0000-0001-7267-4723

\section{REFERENCES}

1 Yang $X, Y u$ Y, Xu J, et al. Clinical course and outcomes of critically ill patients with SARS-CoV-2 pneumonia in Wuhan, China: a singlecentered, retrospective, observational study. Lancet Respir Med 2020;8:475-81.

2 Grasselli G, Zangrillo A, Zanella A, et al. Baseline characteristics and outcomes of 1591 patients infected with SARS-CoV-2 admitted to ICUs of the Lombardy region, Italy. JAMA 2020;323:1574.

3 Han Y, Zhang $\mathrm{H}$, Mu S. Lactate dehydrogenase a risk factor of severe COVID-19 patients. medRxiv 2020. doi:10.1101/2020.03.24.20040162

4 Vidali S, Morosetti D, Cossu E, et al. D-Dimer as an indicator of prognosis in SARS-CoV-2 infection: a systematic review. ERJ Open Res 2020;6. doi:10.1183/23120541.00260-2020. [Epub ahead of print: 13 Jul 2020].

5 van Mirre E, Vrielink GJ, Tjon-a-Tsoi N, et al. Sensitivity and specificity of the high fluorescent lymphocyte count-gate on the Sysmex XE-5000 hematology analyzer for detection of peripheral plasma cells. Clin Chem Lab Med 2011;49:685-8.

6 Oehadian A, Michels M, de Mast Q, et al. New parameters available on Sysmex XE-5000 hematology analyzers contribute to differentiating dengue from leptospirosis and enteric fever. Int $J$ Lab Hematol 2015;37:861-8.

7 Arneth BM, Ragaller M, Hommel K, et al. Novel parameters of extended complete blood cell count under fluorescence flow cytometry in patients with sepsis. J Clin Lab Anal 2014;28:130-5.

8 Clegg A, Young J, Iliffe S, et al. Frailty in elderly people. Lancet 2013;381:752-62.

9 Rockwood K, Andrew M, Mitnitski A. A comparison of two approaches to measuring frailty in elderly people. J Gerontol A Biol Sci Med Sci 2007;62:738-43.

10 Fried LP, Tangen CM, Walston J, et al. Frailty in older adults: evidence for a phenotype. J Gerontol A Biol Sci Med Sci 2001;56:M146-57.

11 Bellelli G, Rebora P, Valsecchi MG, et al. Frailty index predicts poor outcome in COVID-19 patients. Intensive Care Med 2020;46:1634-6.

12 Rockwood K, Song X, MacKnight C, et al. A global clinical measure of fitness and frailty in elderly people. CMAJ 2005;173:489-95.

13 Hewitt J, Carter B, Vilches-Moraga A, et al. The effect of frailty on survival in patients with COVID-19 (cope): a multicentre, European, observational cohort study. Lancet Public Health 2020;5:e444-51.

14 NICE. 2 Admission to critical care / COVID-19 rapid guideline: critical care in adults | Guidance | NICE [Internet]. Available: https://www. nice.org.uk/guidance/ng159/chapter/2-Admission-to-critical-care

15 Guidet B, de Lange DW, Boumendil A, et al. The contribution of frailty, cognition, activity of daily life and comorbidities on outcome in acutely admitted patients over 80 years in European ICUs: the VIP2 study. Intensive Care Med 2020;46:57-69.
16 O'Caoimh R, Kennelly S, Ahern E, et al. Letter to the editor: Covid-19 and the challenges of frailty screening in older adults. J Frailty Aging 2020;9:1-2.

17 Moug S, Carter B, Myint PK, et al. Decision-making in COVID-19 and frailty. Geriatrics 2020;5:30.

18 Miles A, Webb TE, Mcloughlin BC, et al. Outcomes from COVID-19 across the range of frailty: excess mortality in fitter older people. Eur Geriatr Med 2020;11:851-5.

19 Owen RK, Conroy SP, Taub N, et al. Comparing associations between frailty and mortality in hospitalised older adults with or without COVID-19 infection: a retrospective observational study using electronic health records. Age Ageing 2021;50:307-16.

20 Flaatten H, De Lange DW, Morandi A, et al. The impact of frailty on ICU and 30-day mortality and the level of care in very elderly patients ( $\geq 80$ years). Intensive Care Med 2017;43:1820-8.

21 Guidet B, Flaatten $\mathrm{H}$, Boumendil A, et al. Withholding or withdrawing of life-sustaining therapy in older adults $(\geq 80$ years) admitted to the intensive care unit. Intensive Care Med 2018;44:1027-38.

22 Linn BS, Linn MW, Gurel L. Cumulative illness rating scale. J Am Geriatr Soc 1968;16:622-6.

23 Katz S, Ford AB, Moskowitz RW, et al. Studies of illness in the aged. The index of ADL: a standardized measure of biological and psychosocial function. JAMA 1963;185:914-9.

24 Lawton MP, Brody EM. Assessment of older people: selfmaintaining and instrumental activities of daily living. Gerontologist 1969;9:179-86.

25 [French Thoracic Imaging Society proposes a structured chest CT report for patients suspected of COVID-19] [Internet], 2020. Available: http://www.sfrnet.org/portal/actu/news.phtml?id=rc\% 2Forg\%2Fsfrnet\%2Fnws\%2FNews\%2F2020\%2F20200316-155630175

26 Le Gall JR, Lemeshow S, Saulnier F. A new simplified acute physiology score (saps II) based on a European/North American multicenter study. JAMA 1993;270:2957-63.

27 Vincent JL, Moreno R, Takala J, et al. The SOFA (sepsis-related organ failure assessment) score to describe organ dysfunction/ failure. on behalf of the Working group on sepsis-related problems of the European Society of intensive care medicine. Intensive Care Med 1996;22:707-10.

28 Riley RD, Snell KI, Ensor J, et al. Minimum sample size for developing a multivariable prediction model: PART II - binary and time-to-event outcomes. Stat Med 2019;38:30:1276-96.

29 Morley JE, Vellas B, van Kan GA, Abellan van Kan G, et al. Frailty consensus: a call to action. J Am Med Dir Assoc 2013;14:392-7.

30 Muscedere J, Waters B, Varambally A, et al. The impact of frailty on intensive care unit outcomes: a systematic review and meta-analysis. Intensive Care Med 2017;43:1105-22.

31 Kara I, Yildirim F, Zerman A, et al. The impact of frailty on noninvasive mechanical ventilation in elderly medical intensive care unit patients. Aging Clin Exp Res 2018;30:359-66.

32 Fernando SM, Mclsaac DI, Rochwerg B, et al. Frailty and invasive mechanical ventilation: association with outcomes, extubation failure, and tracheostomy. Intensive Care Med 2019;45:1742-52.

33 Fernando SM, Mclsaac DI, Perry JJ, et al. Frailty and associated outcomes and resource utilization among older ICU patients with suspected infection. Crit Care Med 2019;47:e669-76.

34 Bagshaw SM, Stelfox HT, Johnson JA, et al. Long-term association between frailty and health-related quality of life among survivors of critical illness: a prospective multicenter cohort study. Crit Care Med 2015;43:973-82. 